\title{
Preferential coreceptors utilization and cytopathicity by dual-tropic HIV-1 in human lymphoid tissue ex vivo
}

\author{
Svetlana Glushakova, ${ }^{1}$ Yanjie Yi, ${ }^{2}$ Jean-Charles Grivel, ${ }^{1}$ Anjali Singh, ${ }^{2}$ Dominique Schols, ${ }^{3}$ \\ Erik De Clercq, ${ }^{3}$ Ronald G. Collman, ${ }^{2}$ and Leonid Margolis ${ }^{1}$ \\ ${ }^{1}$ Laboratory of Molecular and Cellular Biophysics, National Institute of Child Health and Human Development, National Institutes of Health, \\ Bethesda, Maryland 20892, USA \\ ${ }^{2}$ University of Pennsylvania School of Medicine, Philadelphia, Pennsylvania 19104, USA \\ ${ }^{3}$ Rega Institute for Medical Research, Katholieke Universiteit Leuven, B-3000 Leuven, Belgium \\ Address correspondence to: Ronald Collman, University of Pennsylvannia, 522 Johnson Pavilion, Philadelphia, Pennsylvannia 19104, USA. \\ Phone: (215) 898-0913; Fax: (215) 573-4446; E-mail: collmanr@mail.med.upenn.edu; or Leonid Margolis, National Institute of Child Health, \\ Building 10, Room 10D14, Bethesda, Maryland 20892, USA. Phone: (301) 594-2476; Fax: (301) 480-0857; E-mail: margolis@helix.nih.gov.
}

Received for publication May 21, 1999, and accepted in revised form August 2, 1999.

Many HIV-1 isolates at the late stage of disease are capable of using both CXCR4 and CCR5 in transfected cell lines, and are thus termed dual-tropic. Here we asked whether these dual-tropic variants also use both coreceptors for productive infection in a natural human lymphoid tissue microenvironment, and whether use of a particular coreceptor is associated with viral cytopathicity. We used 3 cloned dual-tropic HIV-1 variants, 89.6 and its chimeras 89-v345.SF and 89-v345.FL, which use both CCR5 and CXCR4 in transfected cell lines. In human lymphoid tissue ex vivo, one variant preferentially used CCR5, another preferentially used CXCR4, and a third appeared to be a true dual-tropic variant. The 2 latter variants severely depleted $\mathrm{CD} 4^{+} \mathrm{T}$ cells, whereas cytopathicity of the virus that used CCR5 only in lymphoid tissue was mild and confined to CCR5 ${ }^{+}$CD4 ${ }^{+}$ $\mathrm{T}$ cells. Thus, (a) HIV-1 coreceptor usage in vitro cannot be unconditionally extrapolated to natural microenvironment of human lymphoid tissue; (b) dualtropic viruses are not homogeneous in their coreceptor usage in lymphoid tissue, but probably comprise a continuum between the 2 polar variants that use CXCR4 or CCR5 exclusively; and (c) cytopathicity toward the general CD4+ $\mathrm{T}$ cell population in lymphoid tissue is associated with the use of CXCR4.

J. Clin. Invest. 104:R7-R11 (1999).

\section{Introduction}

HIV-1 uses CD4 together with coreceptors to enter target cells (reviewed in refs. $1-3)$; the main coreceptors used in vivo are CCR5 (4-7) and CXCR4 (8-10). The former is used by strains that are responsible for person-to-person transmission and are predominant at the early stages of infection. Variants that use CXCR4 frequently appear later in disease, and their emergence is associated with rapid loss of $\mathrm{CD}^{+} \mathrm{T}$ cells $(11,12$; reviewed in ref. 2). In human lymphoid tissue ex vivo, CCR5-using HIV-1 isolates (R5; ref. 13) are mildly cytopathic and deplete less than $20 \%$ of $\mathrm{CD}^{+} \mathrm{T}$ cells $(14,15)$, whereas CXCR4-using HIV-1 isolates (X4; ref. 13) are highly cytopathic and deplete $80-90 \%$ of $\mathrm{CD}^{+} \mathrm{T}$ cells $(14,15)$. The sequences in gp120 that define CCR5 or CXCR4 utilization are sufficient to lar coreceptor is associated with viral cytopathicity.

To study coreceptor use by dual-tropic viruses in the context of human lymphoid tissue, we employed an ex vivo human tonsil system that supports productive HIV-1 infection without exogenous stimulation or activation $(14,15$, 18). In the absence of HIV-1 infection, the relative numbers of $\mathrm{T}$ lymphocytes expressing CCR5 and CXCR4 in this model do not change over almost 2 weeks of incubation (19). We infected these tissues with 3 cloned dual-tropic HIV-1 variants. Strain 89.6 is a primary isolate, and 89-v345.SF and 89-v345.FL are derivatives of 89.6 in which the V3V5 region of gp120 was replaced with corresponding sequences from R5 isolates SF162 or JR-FL, respectively. These chimeras retain the ability to use both CCR5 and CXCR4 for fusion and infection in vitro (20). The use of isogenic viruses that differ only by sequences in gp120 allowed rigorous testing of the effect of coreceptor specificity on viral infection and pathogenicity in a native lymphoid microenvironment. Using this panel of dual-tropic HIV-1 variants, we found that in human lymphoid tissue, one preferentially used CXCR4, another preferentially used CCR5, and a third appeared to be a truly dual-tropic variant. Importantly, we also found that cytopathicity in lymphoid tissue correlated with CXCR4 usage ex vivo.

\section{Methods}

Viruses. We used the dual-tropic R5X4 strain 89.6 and macrophage-tropic (Mtropic) R5 strains SF162 and JR-FL (21, 


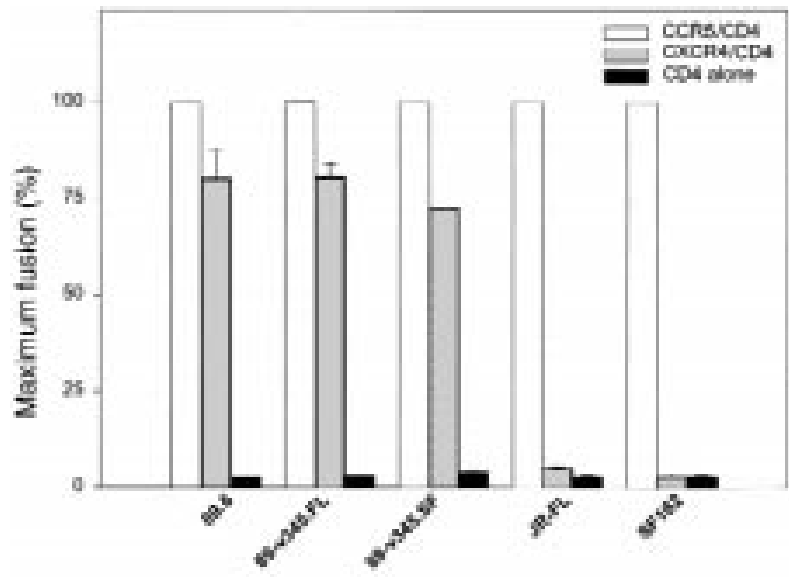

Figure 1

Fusion mediated by dual-tropic env glycoproteins. Effector 293T cells were infected with recombinant vaccinia virus vP11T7gene1, which expresses the T7 RNA polymerase, and then transfected with plasmids encoding the indicated env genes. These were then mixed with quail QT6 cells that had been transfected with plasmids encoding CD4, the indicated coreceptor, and the luciferase reporter gene under control of the T7 promoter. Cells were lysed 16 hours after mixing, and luciferase levels were measured as an indication of cell-cell fusion and reporter gene transactivation. Data represent mean \pm SEM of 3 experiments expressed as percent of maximal luciferase levels observed for each env gene.

22). The 89-v345.FL chimera was generated by introducing into 89.6 a 580 bp $B g l \mathrm{II}-B g l \mathrm{II}$ region of $e n v$ derived from JR-FL, which included the V3-V5 domains. The env fragment was amplified by PCR from genomic DNA of peripheral blood lymphocytes (PBLs) infected with JR-FL, and was cloned into a 3 ' hemi-genome plasmid of 89.6 as described previously (20). The 89v345.SF chimera was generated the same way using a fragment amplified from SF162-infected cells. Recombinant clones were sequenced to verify the inserted JR-FL and SF162 sequences. Infectious viruses were generated by cotransfecting the recombinant $3^{\prime}$ hemi-genome with the $89.65^{\prime}$ hemigenome, followed by an amplification in PBLs as described previously (20).

Coreceptor utilization in vitro. Coreceptor selectivity in vitro was determined by cell-cell fusion and by infection of cells transfected with CD4 and coreceptors. For fusion, the recombinant 3' hemi-genomes, which contain $\mathrm{T} 7$ promoters upstream of env, were transfected into 293T cells infected previously with recombinant vaccinia virus vP11T7gene1, which expresses the T7 RNA polymerase. These were then mixed with target QT6 cells that were previously cotransfected with plasmids encoding CD4, the coreceptor of interest or control vector, and a luciferase reporter gene under control of the T7 promoter. Cell-cell fusion resulted in content mixing and T7 polymerase transactivation of the luciferase reporter gene. Details of this assay have been described previously $(17,20)$. To test coreceptor-mediated infection, QT6 cells were cotransfected with CD4 and coreceptors, then infected with DNAse-treated virus stocks. Three days after infection, cells were lysed, and viral DNA reverse transcription products were detected by PCR amplification followed by Southern blot as described previously (20).

Infection of human lymphoid tissue ex vivo. Human tonsils were obtained from patients undergoing tonsillectomies. Tissues were dissected into approximately 2-mm blocks and incubated on collagen gels at the air-liquid interface. Culture medium was changed every 2-3 days. Detailed culture methods were described previously $(14,18,23)$. Lymphoid tissue blocks were infected using 3-5 $\mu \mathrm{L}$ of clarified virus-containing medium (200-300 ng/mL p24 antigen) applied slowly on top of each tissue block. This inoculum resulted in replication kinetics with maximum p24 antigen production at 12-14 days after infection $(14,18)$, which is the standard time frame for culturing the tissue blocks. RANTES (PeproTech Inc., Rocky Hill, New Jersey, USA) and AMD3100 (obtained from G. Henson, AnorMED, Langley, British Columbia,
Canada) $(24,25)$ were added to the medium 3 hours before infection and were replenished with each medium change. The concentration of p24 gag antigen in the medium was measured by ELISA (Cellular Products Inc., Buffalo, New York, USA; or NCI Laboratories, Frederick, Maryland, USA).

Depletion of $\mathrm{CD}^{+} \mathrm{T}$ cells. Cells were mechanically isolated from tissue blocks on day 14 after infection and stained with a mixture of anti-CD3-PC5, anti-CD4-RD1, and anti-CD8-FITC (Coulter Corp., Miami, Florida, USA) mAb's $(14,19)$. To analyze coreceptor expression on $\mathrm{CD}^{+} \mathrm{T}$ lymphocytes, cells were stained with anti-CD3-FITC and CD4 TriColor (Caltag Laboratories Inc., Burlingame, California, USA), as well as with anti-CXCR4-biotin and anti-CCR5-phycoerythrin (PharMingen, San Diego, California, USA), and analyzed using a FACScalibur (Becton Dickinson Immunocytometry Systems, San Jose, California, USA). To normalize for differences in size and cellularity of tissue blocks, depletion of total $\mathrm{CD}^{+} \mathrm{T}$ cells and cell subsets was expressed as a ratio of the number of these cells to the number of $\mathrm{CD}^{+} \mathrm{T}$ cells that were not affected by the infection (14).

\section{Results}

Human tonsillar tissue maintained ex vivo retains its cellular repertoire (14, 18 ), including the relative presence of CXCR4- and CCR5-expressing cells (19), thus providing targets for HIV-1 variants that use both coreceptors. To test coreceptor utilization and cytopathicity by dual-tropic strains in this model, we used 3 viruses that demonstrate dual CCR5 and CXCR4 utilization in vitro and that are isogenic except for specific env sequences. As shown in Figure 1, env glycoproteins of the parental 89.6 strain and the 89v345.SF and 89-v345.FL chimeras mediated fusion with both CCR5 and CXCR4. All 3 viruses also infected QT6 cells transfected with CD4 and either coreceptor, based on PCR detection of viral reverse transcription products (ref. 20 and data not shown). Also, no differences were revealed in the ability of the 3 dual-tropic strains to use CCR5 or CXCR4 expressed at low levels (data not shown). Thus, these strains represent viruses that can use both coreceptors in vitro.

To test whether these dual-tropic HIV-1 variants could use both CCR5 


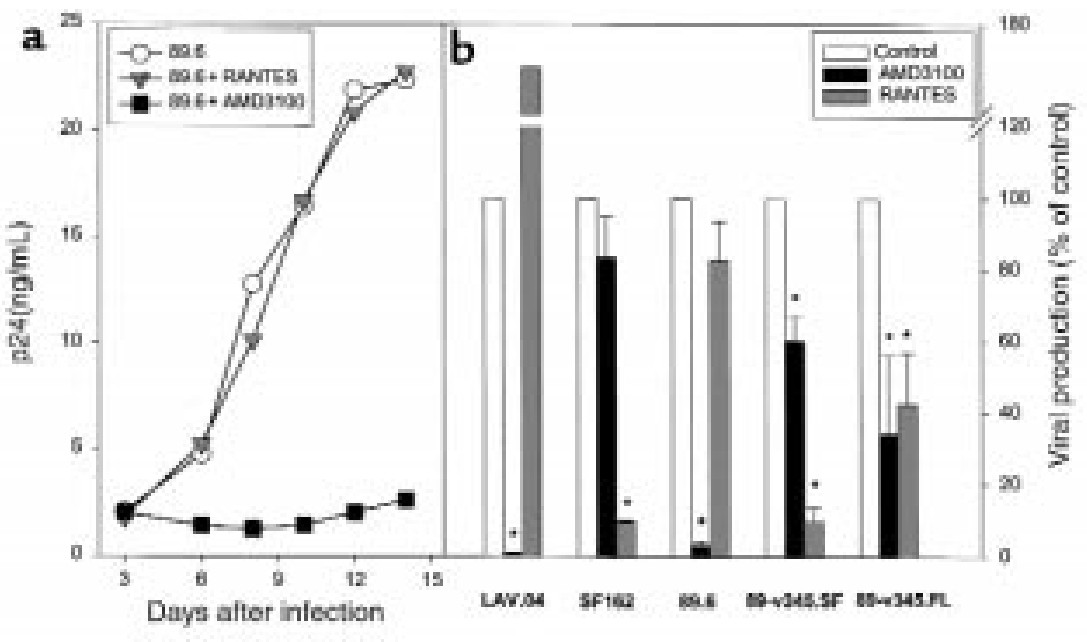

Figure 2

Effect of AMD3100 and RANTES on replication of HIV-1 in human lymphoid tissue ex vivo. Tissue blocks were infected with virus in the absence of blocking agent (control) or after the addition of RANTES $(100 \mathrm{nM})$ or AMD3100 $(1 \mu \mathrm{g} / \mathrm{mL})$, as described in Methods, and were analyzed for p24 in culture medium. (a) Replication kinetics of 89.6. Shown are measurements of p24 antigen in culture medium produced in 20 pooled tissue blocks from an individual donor. (b) Average total production of 24 over 14 days. Mean \pm SEM for $5-8$ experiments with 89.6 and its chimeras and 1-4 experiments with LAV.04 and SF162. The results are expressed as percent of control-infected tissue. * Significant inhibition of viral production $(P<0.05)$.

and CXCR4 in human lymphoid tissue ex vivo, we used CCR5 or CXCR4 ligands to block viral entry. We used the CC chemokine RANTES to inhibit entry through CCR5 (26), and the small-molecular-weight bicyclam AMD3100 to block entry through CXCR4 $(24,25)$. The blocking potentials of these agents in a complex microenvironment of lymphoid tissue were first tested using HIV-1 isolates restricted to each specific coreceptor. Figure 2 shows that AMD3100 almost completely blocked ex vivo infection with the prototype X4 HIV-1 isolate LAV.04 in human lymphoid tissue. As expected, RANTES did not inhibit LAV.04 (Figure 2b), but, rather, appeared to enhance infection, as has been previously reported $(23,27,28)$. The R5 prototype SF162 was affected in the opposite way: CXCR4 antagonist AMD3100 inhibited SF162 infection only marginally (statistically insignificant, $P=0.2$ ), whereas CCR5 ligand RANTES did so in a significant way (Figure 2b). Thus, these agents retain their coreceptor-specific HIV-inhibiting activity in the native lymphoid microenvironment.

Based on these findings, we used AMD3100 and RANTES to test whether dual-tropic viruses use both CXCR4 and CCR5 in human lymphoid

Figure 3 T lymphocytes. Data from an individual experiment.
On the other hand, the dual-tropic strain 89 -v345.SF gave different results (Figure 2b). Replication of this virus was almost completely blocked by RANTES. In contrast, inhibition of replication of this virus by AMD3100 was much less efficient, although statistically significant (Figure 2b). Based on coreceptor use in lymphoid tissue ex vivo, 89-v345.SF was much closer to R5 isolate SF162 and unlike 89.6, which was itself more similar to $\mathrm{X} 4$ isolates (e.g., LAV.04). The third dual-tropic variant tested, 89-v345.FL, was almost equally sensitive to AMD3100 and RANTES (Figure 2b), although neither agent alone completely blocked infection. Thus, 89-v345.FL appears to use both coreceptors in lymphoid tissue. Therefore, while the 3 HIV- 1 isolates all exhibited R5X4 dual-coreceptor utilization in vitro, in the complex microenvironment of human lymphoid tissue, some behave as "more X4," while others are "more R5."

In previous studies, we showed that CXCR4-restricted X4 isolates severely depleted $\mathrm{CD} 4^{+} \mathrm{T}$ cells in ex vivo infected human lymphoid tissues, whereas CCR5-restricted R5 isolates depleted $\mathrm{CD}^{+}{ }^{+} \mathrm{T}$ cells only mildly. Therefore, we next addressed whether coreceptor usage by these variants in tissue infection correlated with cytopathicity. We assessed $\mathrm{CD}^{+} \mathrm{T}$-cell depletion in human tonsillar tissues infected ex vivo

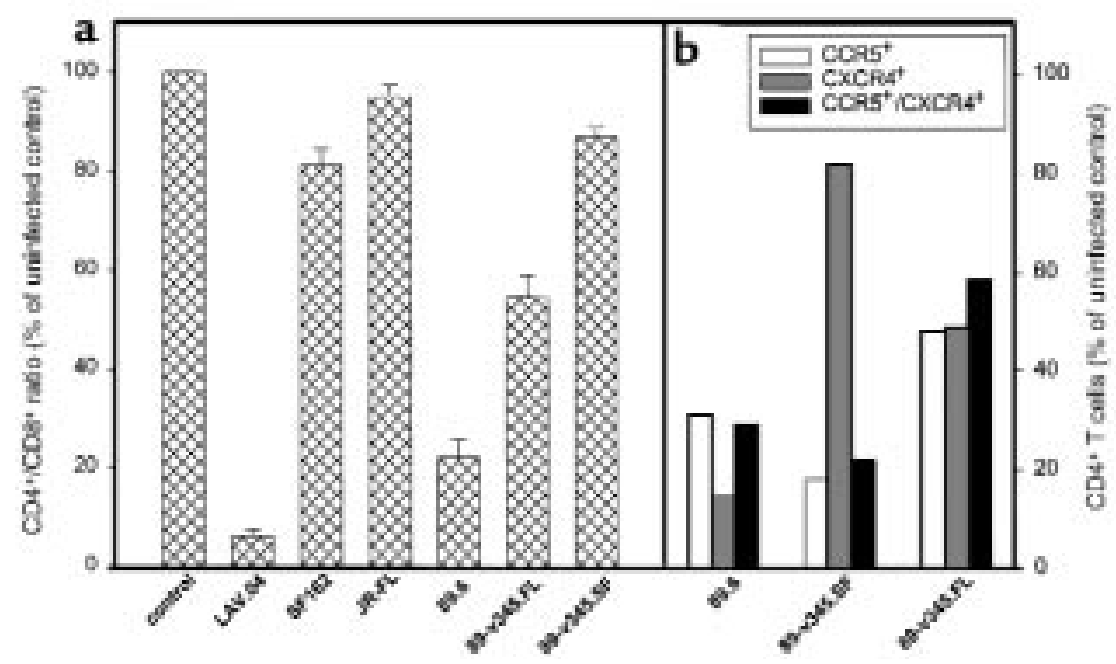

Depletion of CD4 ${ }^{+} \mathrm{T}$ cells in human lymphoid tissue infected ex vivo with HIV-1. Lymphocytes were isolated on day 14 after infection, stained with fluorescent antibodies, and analyzed using flow cytometry. The results are expressed as percent of total $C D 4^{+} \mathrm{T}$ cells or their subsets relative to matched uninfected control. (a) Depletion of total CD4 ${ }^{+}$T lymphocytes. Mean \pm SEM for $15-23$ experiments with tissues from individual donors. (b) Depletion of CXCR4 $4^{+} / \mathrm{CD} 4^{+}$and $C \mathrm{CR} 5^{+} / \mathrm{CD}^{+}$ 
with 89.6, 89-v345.SF, and 89-v345.FL, as well as with the R5 strains SF162 and JR-FL, on day 14 after infection. These results are presented in Figure 3a. Strain 89.6 was highly cytopathic, depleting $\mathrm{CD}^{+} \mathrm{T}$ cells to $22 \pm 4 \%$ of control values $(n=21)$, and thus resembled X4 variants in terms of cytopathicity. In contrast, 89-v345.SF was mildly cytopathic, with $87 \pm 2 \%(n=23)$ of $\mathrm{CD}^{+} \mathrm{T}$ cells remaining in the infected tissue. The limited cytopathicity of 89v345.SF resembled that of all tested R5 isolates (19) and was consistent with its principal use of CCR5 in lymphoid tissues. Strain 89-v345.FL demonstrated an intermediate level of cytopathicity, resulting in $54 \pm 4 \%(n=19)$ of the $\mathrm{CD}^{+}$ $\mathrm{T}$ cells remaining in infected lymphoid tissues. Thus, high viral cytopathicity of dual-tropic HIV-1 isolates in lymphoid tissue correlated with preferential utilization of CXCR4 in this environment.

One major reason that X4 HIV-1 variants may be more cytopathic than R5 variants in lymphoid tissue is that the latter kills only CCR5 ${ }^{+}$cells, which constitute less than $15 \%$ of the total $\mathrm{CD}^{+}$ $\mathrm{T}$ cells, whereas $\mathrm{CXCR} 4^{+}$cells make up more than $80 \%$ of the $\mathrm{CD}^{+} \mathrm{T}$-cell population (19). We therefore analyzed the cytopathicity of the 89-v345.SF variant. Figure 3b shows that, like SF162 and other R5 variants (14), 89-v345.SF depleted only $\mathrm{CCR}^{+} / \mathrm{CD}^{+}{ }^{+} \mathrm{T}$ cells, leaving the population of $\mathrm{CXCR} 4^{+} / \mathrm{CD}^{+}{ }^{+} \mathrm{T}$ cells intact. This result is in agreement with the preferential CCR5 usage by this virus, as demonstrated by its efficient inhibition with RANTES. In contrast, 89.6 and 89-v345.FL depleted all $\mathrm{CD}^{+}$T-cell subsets (Figure $3 \mathrm{~b}$ ). Whether the depletion of CCR5 ${ }^{+} / \mathrm{CD}^{+}$ T cells by the $89.6 \mathrm{HIV}-1$ isolate represents a bystander effect or whether these cells express CXCR4 at a level undetectable by flow cytometry, but sufficient for X4 variants to recognize these cells, remains to be studied (19). Whatever the mechanism of $\mathrm{CD}^{+}{ }^{+} \mathrm{T}$ cell depletion, both 89.6 and 89v345.FL are similar in this respect to X4 isolates that use CXCR4 as the single coreceptor (19).

\section{Discussion}

In this study we analyzed $3 \mathrm{HIV}-1$ variants derived from 89.6 that use both CCR5 and CXCR 4 coreceptors in vitro, and have shown that in human lymphoid tissue they exhibit diverse behavior. One preferentially used CXCR4 for infection and was highly cytopathic; another appeared to use both CXCR4 and CCR5 and showed intermediate cytopathicity; and the third used CCR5 preferentially and exhibited mild cytopathicity because it eliminated only the minority of $\mathrm{CD}^{+} \mathrm{T}$ cells that express CCR5.

The first conclusion from these experiments is that HIV-1 coreceptor usage in vitro cannot necessarily be extrapolated to human lymphoid tissue. This is exemplified by 89.6, which efficiently uses both CCR 5 and CXCR4 in primary and transfected cells in vitro $(17,29)$ but uses CXCR4 exclusively in lymphoid tissue ex vivo. Similarly, 89v345.SF uses both coreceptors in vitro but uses mainly CCR5 ex vivo. This restricted utilization ex vivo occurs despite the fact that, as we showed earlier (19) and confirmed here, both CXCR4 and CCR5 are expressed on the cell surface of $\mathrm{CD}^{+} \mathrm{T}$ lymphocytes in human lymphoid tissue. Distinctions between coreceptor use in transfected cells and in primary PBMCs was recently highlighted by Zhang and Moore (30). Thus, coreceptor tropism of HIV1 is even more restricted in human lymphoid tissue than in PBMCs, making some dual-tropic strains essentially single coreceptor-dependent. The reason for this is not clear, but may reflect different levels of coreceptor expression on target cells in lymphoid tissue compared with cells cultured in vitro. Because the viruses tested were isogenic, except for a 580-bp V3-V5 region of env, our results further show that these differences in coreceptor utilization for productive infection ex vivo are linked to the envelope glycoprotein.

Strain 89.6 is a naturally occurring primary isolate, whereas the other dualtropic viruses studied are recombinants. We focused on this panel because they are isogenic (except for $e n v$ ), so they not only highlight the critical differences between coreceptor selectivity in vitro and in the native lymphoid environment, but also show that env is the molecular determinant. One question is whether other naturally occurring dual-tropic strains that use both coreceptors in vitro are also restricted to CXCR4 ex vivo, like 89.6, or whether they use CCR5, either as their principal coreceptor, like 89v345.SF, or along with CXCR4, like 89v345.FL. In preliminary studies, we have found that the dual-tropic isolate
DH12 (31), which also uses both CCR5 and CXCR4 in vitro (32), is not blocked by AMD3100 or RANTES in lymphoid tissue, even though these agents block DH12 entry through CXCR4 and CCR5, respectively, in transfected cell lines (data not shown). This suggests that DH12 differs from 89.6 and is not restricted to CXCR4 ex vivo. It remains to be determined if other naturally occurring strains are dual-tropic in vitro but are restricted to CCR5 ex vivo, like 89-v345.SF.

An important implication of these results is that the $\mathrm{R} 5 \mathrm{X} 4$ viruses are not a homogeneous group in coreceptor usage, but probably comprise a continuum between the 2 polar variants, R5 and X4. Additional studies involving a broad survey of naturally occurring cloned dual-tropic isolates will be required to determine the relative frequency of CXCR4-restricted, CCR5restricted, and dual-coreceptor use in lymphoid tissue.

Another conclusion of this study is that viral cytopathicity toward the general $\mathrm{CD}^{+}{ }^{+} \mathrm{T}$-cell population in lymphoid tissue is closely associated with use of CXCR4. We previously showed this to be the case for HIV-1 strains restricted to single coreceptors (14-16, 19). Our present study provides evidence that this rule is valid for both single-tropic and dual-tropic HIV-1 isolates.

\section{Acknowledgments}

This work was supported in part by the NASA/NIH Center for Three Dimensional Tissue Culture and by NIH grants HL-58004 and AI-35502 to R.G. Collman. We are grateful to David Lowry for assistance and Joshua Zimmerberg for support and encouragement.

\footnotetext{
1. Moore, J.P., Trkola, A., and Dragic, T. 1997. Coreceptors for HIV-1 entry. Curr. Opin. Immunol. 9:551-562.

2. Berger, E.A., Murphy, P.M., and Farber, M.J. 1999. Chemokine receptors as HIV-1 coreceptors: roles in viral entry, tropism and disease. Annu. Rev. Immunol. 17:657-700.

3. Locati, M., and Murphy, P.M. 1999. Chemokines and chemokine receptors: biology and clinical relevance in inflammation and AIDS. Annu. Rev. Med. 50:425-440.

4. Dean, M., et al. 1996. Genetic restriction of HIV1 infection and progression to AIDS by a deletion allele of the CKR5 structural gene. Hemophilia Growth and Development Study, Multicenter AIDS Cohort Study, Multicenter Hemophilia Cohort Study, San Francisco City Cohort, ALIVE Study. Science. 273:1856-1862.

5. Huang, Y., et al. 1996. The role of a mutant CCR5 allele in HIV-1 transmission and disease progression. Nat. Med. 2:1240-1243.

6. Liu, R., et al. 1996. Homozygous defect in HIV-1
} 
coreceptor accounts for resistance of some multiply exposed individuals to HIV-1 infection. Cell. 86:367-377.

7. Samson, M., Labbe, O., Mollereau, C., Vassart, G., and Parmentier, M. 1996. Molecular cloning and functional expression of a new human CCchemokine receptor gene. Biochemistry. 35:3362-3367.

8. Bjorndal, A., et al. 1997. Coreceptor usage of primary human immunodeficiency virus type 1 isolates varies according to biological phenotype. $J$. Virol. 71:7478-7487.

9. Connor, R.I., Sheridan, K.E., Ceradini, D., Choe, S., and Landau, N.R. 1997. Change in coreceptor use correlates with disease progression in HIV-1-infected individuals. J. Exp. Med. 185:621-628.

10. Simmons, G., et al. 1997. Potent inhibition of HIV1 infectivity in macrophages and lymphocytes by a novel CCR5 antagonist. Science. 276:276-279.

11. Tersmette, M., et al. 1989. Association between biological properties of human immunodeficiency virus variants and risk for AIDS and AIDS mortality. Lancet. 1:983-985.

12. Zhu, T., et al. 1993. Genotypic and phenotypic characterization of HIV-1 patients with primary infection. Science. 261:1179-1181.

13. Berger, E.A., et al. 1998. A new classification for HIV-1. Nature. 391:240.

14. Glushakova, S., Baibakov, B., Zimmerberg, J., and Margolis, L. 1997. Experimental HIV infection of human lymphoid tissue: correlation of CD4+ T cell depletion and virus syncytium-inducing/nonsyncytium-inducing phenotype in histoculture inoculated with laboratory strains and patient isolates of HIV type 1. AIDS Res. Hum. Retroviruses. 13:461-471.

15. Glushakova, S., Baibakov, B., Margolis, L.B., and Zimmerberg, J. 1995. Infection of human tonsil histocultures: a model for HIV pathogenesis. Nat. Med. 1:1320-1322.

16. Penn, M.L., Grivel, J.C., Schramm, B., Goldsmith, M.A., and Margolis, L. 1999. CXCR4 utilization is sufficient to trigger CD4+ T cell depletion in HIV1-infected human lymphoid tissue. Proc. Natl. Acad. Sci. USA. 96:663-668.

17. Doranz, B.J., et al. 1996. A dual-tropic primary HIV-1 isolate that uses fusin and the betachemokine receptors CKR-5, CKR-3, and CKR-2b as fusion cofactors. Cell. 85:1149-1158.

18. Glushakova, S., et al. 1998. Evidence for the HIV-1 phenotype switch as a causal factor in acquired immunodeficiency. Nat. Med. 4:346-349.

19. Grivel, J.C., and Margolis, L.B. 1999. CCR5- and CXCR4-tropic HIV-1 are equally cytopathic for their T-cell targets in human lymphoid tissue. Nat. Med. 5:344-346.

20. Smyth, R.J., Yi, Y., Singh, A., and Collman, R.G. 1998. Determinants of entry cofactor utilization and tropism in a dualtropic human immunodeficiency virus type 1 primary isolate. J. Virol. 72:4478-4484.

21. Koyanagi, Y., et al. 1987. Dual infection of the central nervous system by AIDS viruses with distinct cellular tropisms. Science. 236:819-822.

22. Cheng-Mayer, C., Weiss, C., Seto, D., and Levy, J.A 1989. Isolates of human immunodeficiency virus type 1 from the brain may constitute a special group of the AIDS virus. Proc. Natl. Acad. Sci. USA. 86:8575-8579.

23. Margolis, L.B., Glushakova, S., Grivel, J.C., and Murphy, P.M. 1998. Blockade of CC chemokine receptor 5 (CCR5)-tropic human immunodeficiency virus- 1 replication in human lymphoid tissue by CC chemokines. J. Clin. Invest 101:1876-1880

24. Schols, D., et al. 1997. Inhibition of T-tropic HIV strains by selective antagonization of the chemokine receptor CXCR4. J. Exp. Med. 186:1383-1388.

25. Donzella, G.A., et al. 1998. AMD3100, a small molecule inhibitor of HIV-1 entry via the CXCR4 coreceptor. Nat. Med. 4:72-77.

26. Cocchi, F., et al. 1995. Identification of RANTES, MIP-1 alpha, and MIP-1 beta as the major HIVsuppressive factors produced by CD8+ T cells. Science. 270:1811-1815.

27. Kinter, A., et al. 1998. CC-chemokines enhance the replication of T-tropic strains of HIV-1 in CD4(+) T cells: role of signal transduction. Proc. Natl. Acad. Sci. USA. 95:11880-11885.

28. Gordon, C.J., et al. 1999. Enhancement of human immunodeficiency virus type 1 infection by the CC-chemokine RANTES is independent of the mechanism of virus-cell fusion. J. Virol. 73:684-694.

29. Yi, Y., Rana, S., Turner, J.D., Gaddis, N., and Collman, R.G. 1998. CXCR-4 is expressed by primary macrophages and supports CCR5-independent infection by dual-tropic but not T-tropic isolates of human immunodeficiency virus type 1. J. Virol. 72:772-777.

30. Zhang, Y., and Moore, J.P. 1999. Will multiple coreceptors need to be targeted by inhibitors of human immunodeficiency virus type 1 entry? J. Virol. 73:3443-3448.

31. Shibata, R., et al. 1995. Isolation and characterization of a syncytium-inducing, macrophage/T-cell line-tropic human immunodeficiency virus type 1 isolate that readily infects chimpanzee cells in vitro and in vivo. J. Virol. 69:4453-4462.

32. Cho, M.W., et al. 1998. Identification of determinants on a dual tropic human immunodeficiency virus type 1 envelope glycoprotein that confer usage of CXCR4. J. Virol. 72:2509-2515. 